\title{
Utility of Coronary Artery Calcium Scores in Predicting Risk of Subclinical Cardiovascular Atherosclerotic Disease: An Analysis of Limitations to its Adoption With Policy Recommendations
}

\author{
Muhammad M. Ali ${ }^{1}$, Sajjad Gul ${ }^{2}$, Muzna Naqvi ${ }^{3}$, Laila Hakam ${ }^{3}$, Asad Inayat ${ }^{4}$, Sameer Saleem ${ }^{5}$, \\ Mounika Polavarpu ${ }^{6}$, Mubbasher A. Syed ${ }^{7}$ \\ 1. Internal Medicine, University of Toledo, Toledo, USA 2. Internal Medicine, Order of St. Francis - St. Francis Medical \\ Center, Peoria, USA 3. Internal Medicine, Banner Health, Phoenix, USA 4. Internal Medicine, Khyber Teaching Hospital, \\ Peshawar, PAK 5. Internal Medicine, Presence Health St Joseph Hospital, Chicago, USA 6. Public Health, University of \\ Toledo, Toledo, USA 7. Interventional Cardiology, Banner Health, Pheonix, USA
}

Corresponding author: Mubbasher A. Syed, masyed84@hotmail.com

\begin{abstract}
This survey-based analysis aims to highlight key limitations to a wider adoption of coronary artery calcium (CAC) scoring as a means of screening asymptomatic individuals for atherosclerotic cardiovascular disease. The need for a screening tool that adds objective anatomical information to historically established risk scores in the aforementioned population has been met by this imaging modality. Despite that, there has been a hesitance towards frequent usage of these scans. Within the pre-set sampling frame of the University of Toledo, a convenience sampling technique was used to reach out to 60 health care providers. The resultant responses were analyzed and discussed. In addition to identifying patients who need to be worked up further, CAC scans can also help re-stratify patients within-risk groups and inform decision-making regarding the use of lipid-lowering therapies. The public health impact of a greater but appropriate utilization of this diagnostic tool will be impactful. This analysis seeks to better understand real-life obstacles to a wider adoption of these scans and attempts to lay out policy recommendations to address these issues.
\end{abstract}

Review began 04/05/2021 Review ended 04/21/2021 Published 04/23/2021

\section{(c) Copyright 2021}

Ali et al. This is an open access article distributed under the terms of the Creative Commons Attribution License CC-BY 4.0., which permits unrestricted use, distribution, and reproduction in any medium, provided the original author and source are credited.

Categories: Cardiology, Internal Medicine, Preventive Medicine

Keywords: coronary artery calcium scans, atherosclerosis, screening

\section{Introduction}

It is well established that cardiovascular disease is the leading cause of death in the United States and globally, with coronary artery disease (CAD) accounting for more than half of all such deaths [1]. Nearly a quarter of the patients who present with acute myocardial infarctions never experience warning signs or symptoms prior to the incident [2]. The ability to identify patients who remain asymptomatic leading up to such life-threatening events is the holy grail for researchers and physicians alike.

Cardiovascular risk scores have been historically used to fill this void, with the commonest employed tool being the Framingham Risk Score, often abbreviated as FRS [3]. A retrospective analysis by Wilson et al. in 1998 elucidated that the accuracy of FRS is $75 \%$ at best, which fails nearly a quarter of the patients [4]. The ability to characterize coronary artery calcium (CAC) by computed tomography (CT) and using that to predict the risk of future cardiovascular events has been the focus of much scientific work recently $[5,6]$. The presence of calcium in coronaries was historically labelled as a passive process resulting from senility, but the modern pathophysiologic understanding sees it as an active process that involves multiple mediators [68].

The understanding that calcification within atherosclerotic plaques of coronary arteries is a highly specific process that can be non-invasively detected and screened for led to multiple large population-based studies in the United States and European Countries as far back as 2002 [9,10]. Most databanks concurred with the initial reports of Agatston et al. published back in 1990 and furnished strong evidence of an association between detection of calcium in coronaries and future major adverse cardiovascular events in asymptomatic patients [11-13]. It is hence of high screening value to identify those patients who have a lower risk within the intermediate-risk category of asymptomatic patients based on the conventional tools, and CAC scans can fill this gap. Despite the established role and benefit of these scans as screening tools, they are scarcely used in everyday practice [14-18]. Hence, to better understand the limitations towards a wider adoption of these scans, a survey-based analysis was designed and conducted.

\section{Materials And Methods}


One of the cardinal objectives was to integrate input from health care workers who commonly participate in deciding whether to conduct CAC scoring as a screening procedure. The sampling frame was set to include providers within the faculty and academia of the University of Toledo. The aim of the survey was to extract opinions on various facets of CAC screening from all quarters including primary care physicians (PCPs), cardiology fellows, general cardiologists, and interventional cardiologists. A survey was chosen to conduct the interviews in a systematic manner to ensure that the same question was pitched with similar options to all participants to minimize bias. The need to conduct the analysis in a systematic manner, as well as the increasing concerns with in-person interactions during the COVID 19 pandemic, culminated in the decision to use a web-based online questioning tool to request opinions from participating health care workers. A well-known survey designing website called surveymonkey.com was utilized to design a simple questionnaire comprising eight close-ended questions. The questions were pointed, touched upon most aspects being investigated, and were brief and few to ensure that filling the survey takes under 3 minutes to complete. Within the pre-set sampling frame of the University of Toledo, a convenience sampling technique was used to reach out to 60 health care providers including 25 primary care and family practice physicians, 15 general cardiology practitioners (including three nurse practitioners that work closely with them), 10 interventional cardiologists, and 10 cardiology fellows in training (nine general cardiology and one interventional cardiology fellow). It can also be argued that the final sample was a volunteer sample since respondents volunteered to respond. All invitees were emailed requesting them to fill out the survey and explaining the subject and brevity of the questionnaire. The resultant responses were arranged into tables and figures using the online analytic tools on the web-based survey engine.

\section{Results}

A response rate of $50 \%$ ( 30 responses for 60 invitations) was generated by the survey. The breakdown for various categories of health care providers responding to the survey is given in Figure 1, which shows that nearly half the respondents were PCPs. Figure 2 demonstrates that $86.6 \%(26 / 30)$ respondents agreed that CAC scans had a role to play in the management of asymptomatic patients with a risk for CAD. Figure 3 shows the response to the third question in the survey, which queried the default strategy used by these providers to screen asymptomatic patients for coronary disease. While $56.6 \%$ providers chose ASCVD-RE (Atherosclerotic Cardiovascular Disease Risk Estimator) alone, another 30\% of respondents reported relying on a combination of the aforementioned risk estimator and CAC screening.

What best fits your role as a healthcare provider in adult medicine?

\begin{tabular}{|c|c|}
\hline ANSWER CHOICES & RESPONSES \\
\hline Primary Care Provider / Family Physician & $41.94 \%$ \\
\hline General / Non-invasive Cardiologist & $22.58 \%$ \\
\hline Interventional Cardiologist & $12.90 \%$ \\
\hline Cardiology Fellow in Training & $22.58 \%$ \\
\hline TOTAL & \\
\hline
\end{tabular}

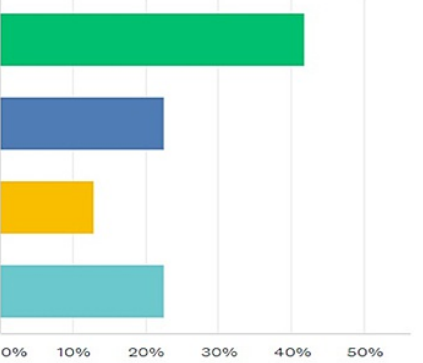

FIGURE 1: Identifying survey respondents according to their role to better delineate the sample 


\section{Cureus}

Do you think there is a role for Coronary Artery Calcium Scoring [CAC Scoring] for management of asymptomatic patients at risk for coronary artery disease?

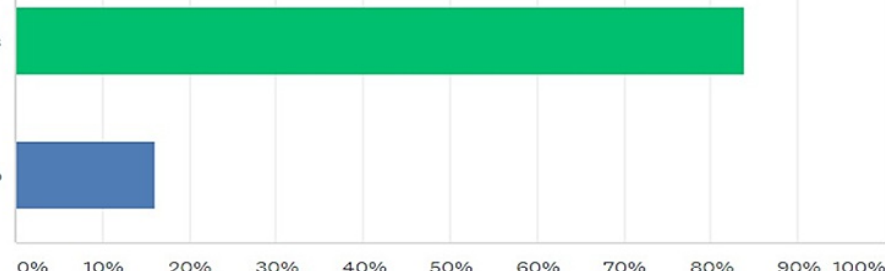

FIGURE 2: Querying the role of CAC scoring as a screening tool for coronary atherosclerosis in asymptomatic patients

CAC, coronary artery calcium

What is your default strategy when screening asymptomatic patients for sub-clinical cardiovascular atherosclerosis?

ANSWER CHOICES
Framingham Risk Score [FRS]
ASCVD Risk Estimator / Revised Pooled Cohort Equation [ASCVD-RE]
CAC Scoring
hSCRP [high sensitivity CRP]
FRS +/-CAC Scoring
ASCVD-RE +/-CAC Scoring
FRS +/-hSCRP
ASCVD-RE +/- hSCRP
TOTAL

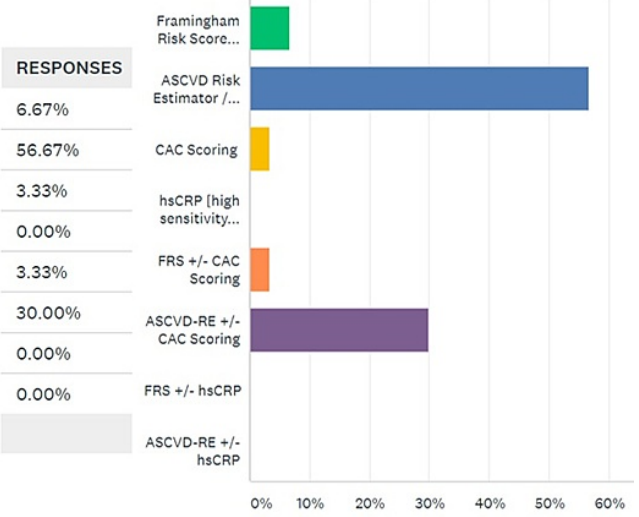

FIGURE 3: Surveying the default strategy for screening asymptomatic individuals for coronary atherosclerosis

In the fourth question, respondents were asked to choose appropriate scenarios to order a CAC scan for atherosclerotic screening. Figure 4 shows that most providers agreed that CAC scores may add value to the evaluation in low-risk subsets with a family history of premature CAD (63.3\% of providers) and intermediate-risk subsets that have a 10-20\% risk of CAD based on risk factor estimations (56.6\% of the providers). Only $16.6 \%$ agreed with screening low-risk women, while $30 \%$ of providers deemed it appropriate to screen high-risk asymptomatic patients. In response to the fifth question on the survey, most respondents (46.6\%) agreed that downstream testing costs are decreased if CAC scans are performed appropriately (Figure 5). 


\section{Cureus}

Which of the following patients would you deem appropriate for a CAC Scoring Scan? [Check all that apply]

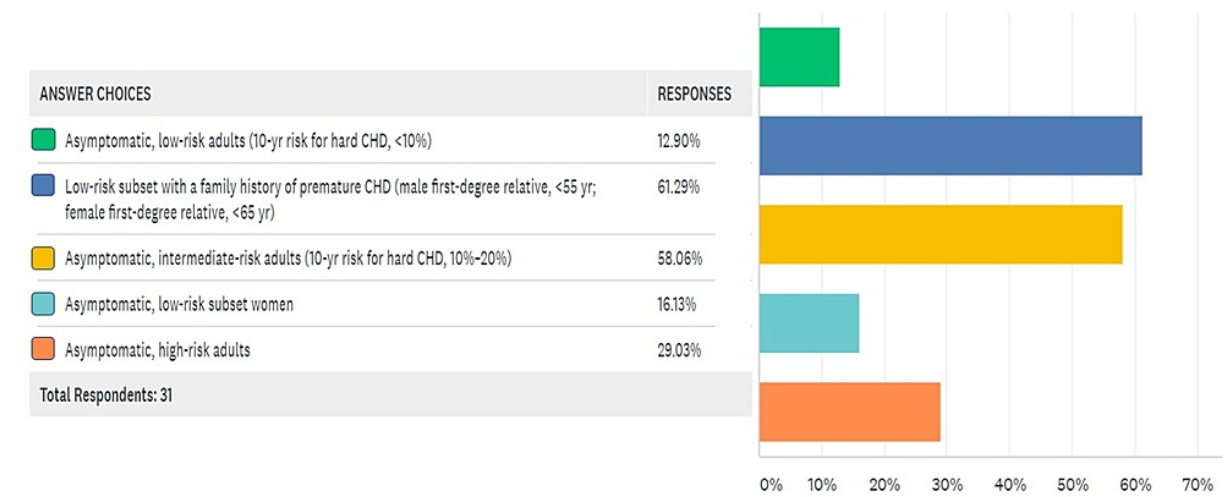

FIGURE 4: The challenge of appropriate patient selection for ordering CAC scans

CAC, coronary artery calcium

The use of any new test may potentially lead to increased downstream healthcare use, so-called layered testing. How does CAC Scan impact downstream medical costs in your opinion?

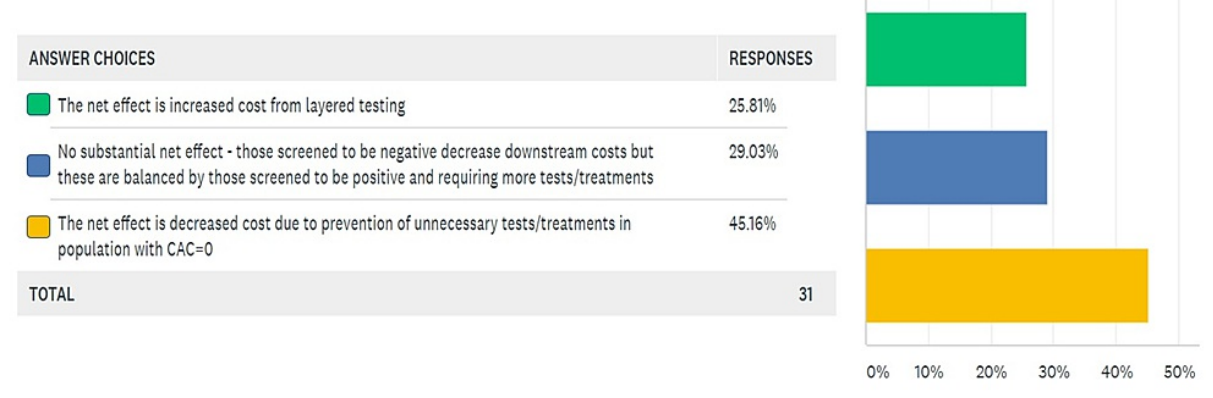

FIGURE 5: Opinions on downstream testing with CAC scans

CAC, coronary artery calcium

When asked if benefit of an indicated CAC scan outweighed a 1-mSv exposure to radiation and the associated carcinogenic potential, in the sixth question on the survey, a larger number of providers believed that the benefit was enough to outweigh the risk, but 40\% (12 of 30) respondents felt otherwise, as shown in Figure 6. The seventh question asked respondents about the frequency with which they faced issues with insurance approval for CAC scoring scans. Only three of 30 respondents reported to have "never" encountered such problems, while 70\% of respondents answered either "always", "usually", or "sometimes" (Figure 7). In the same vein, the last and eighth question on the survey was meant to investigate the issues perceived as major obstacles to a greater adoption of CAC scans as a screening modality among the providers responding to the survey. Of the respondents, $70 \%$ agreed that lack of enthusiasm/adoption was the biggest obstacle to higher usage followed by a small target population ( $40 \%$ respondents) and insurance/cost-related issues for $38.3 \%$ respondents. These findings are summarized in Figure 8. 


\section{Cureus}

The radiation exposure for a CAC scan is roughly $1 \mathrm{mSv}$, similar to that of a bilateral mammogram, and equivalent to $\approx 120$ extra days of background environmental radiation exposure. Do you think this small but certain increase in lifetime risk of cancer outweighs the benefit of timely detection of predisposition to cardiovascular morbidity and/or avoiding unnecessary treatments?

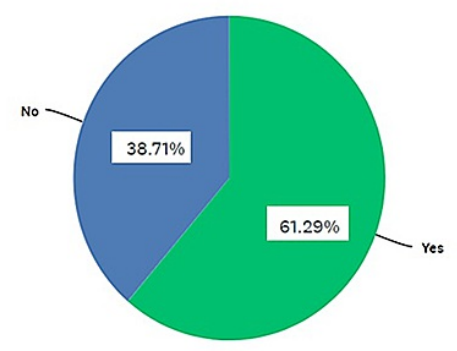

FIGURE 6: Survey respondents on the risk of radiation exposure with CAC scans

CAC, coronary artery calcium

How often do you experience issues with insurance approval for CAC Scans?

\begin{tabular}{|c|c|}
\hline ANSWER CHOICES & RESPONSES \\
\hline$\square$ Always & $9.68 \%$ \\
\hline Usually & $25.81 \%$ \\
\hline Sometimes & $32.26 \%$ \\
\hline Rarely & $19.35 \%$ \\
\hline$\square \quad$ Never & $12.90 \%$ \\
\hline TOTAL & \\
\hline
\end{tabular}

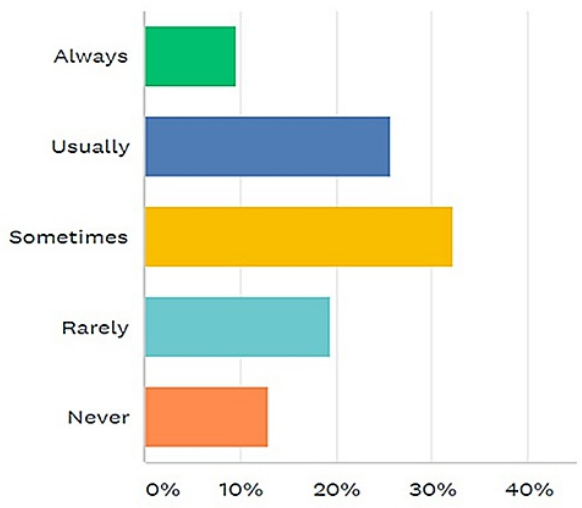

FIGURE 7: The frequency of challenges from insurance companies regarding approval of CAC scans

CAC, coronary artery calcium

What do you think is the biggest limitation to higher adoption of CAC Scans as a technology? [Check all that apply]
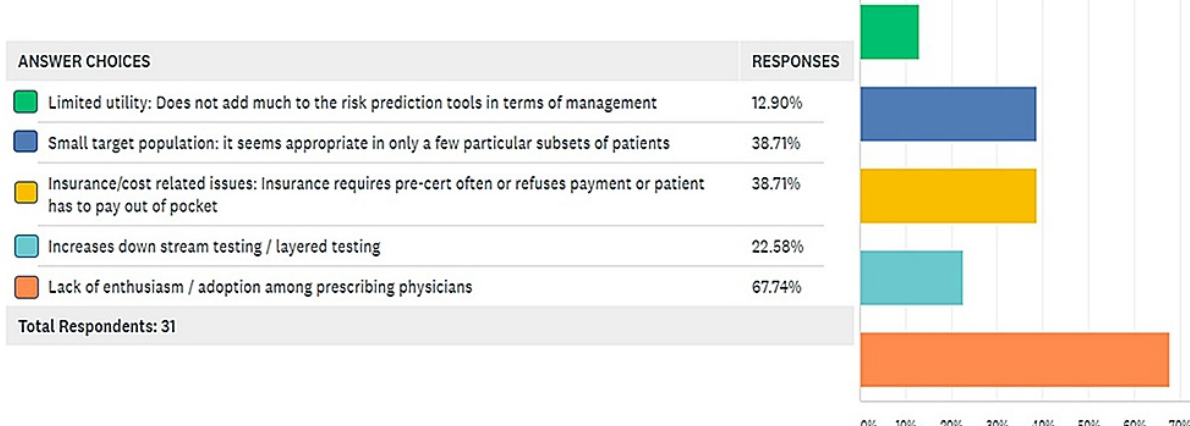

FIGURE 8: Stratifying the limitations to a wider adoption of CAC scans as a screening tool

CAC, coronary artery calcium 


\section{Discussion}

After the detailed assessment of the scientific rationale of using coronary calcium scoring modalities as a screening test for asymptomatic patients with either intermediate-risk profile or a family history of premature CAD, and its utility in these subsets, the survey results above can help identify a pattern of modifiable obstacles that need to be rectified.

The survey was able to elicit response from many stakeholders in the CAC scanning paradigm. This was partly due to the simple and concise design of the survey in accordance with the findings of Liu and Wronski that assert that long and detailed surveys rarely achieve a good response rate [19]. Most respondents were PCPs since 25 out of 60 surveys were sent out to that group of providers, which have a central role in screening asymptomatic patients. Nearly $87 \%$ of respondents agreed that CAC scans had a role in risk management for asymptomatic patients, but a majority (56.6\%) chose the ASCVD-RE alone, a risk estimation tool that is rooted in risk factor analysis, over a combination of ASCVD-RE and CAC scan (30\%) [20]. Evidence suggests that this approach is less accurate [17]. Similarly, the response to question 4 (Figure 4) shows that even in providers that are routinely seeing adult patients in whom a CAC scan maybe indicated, there is a lot of intellectual dissonance. While multiple studies including the landmark publication by Lakoski et al. have validated the indication to screen low-risk women given the atypical symptomology for coronary disease in this gender, only $16.6 \%$ agreed with such a strategy [21]. Moreover, a staggering $30 \%$ of providers deemed it appropriate to screen high-risk asymptomatic patients, which has been shown to be inefficient based on the Bayesian theorem [22].

Another common theme surrounding the academic discussion on increased adoption of CAC scanning has been the financial and radiological costs of this screening tool. It was initially proposed that routinely conducting CAC scans in asymptomatic adults would increase downstream testing and hence overall cost. Some preliminary studies that were critiqued for inappropriate patient selection suggested an unnecessary increase in downstream testing costs [23]. A randomized trial by Rozanski et al. in 2011 demonstrated that compared to no scanning, CAC scanning was associated with much better coronary disease risk factor control without increasing downstream medical testing [24]. Similarly, another commonly cited risk associated with routine use of CAC screening is the radiation exposure associated with it. Budoff et al. established that electron beam CT (EBCT) has an effective radiation dose of 0.7 to $1.0 \mathrm{mSv}$ in men and 0.9 to $1.3 \mathrm{mSv}$ in women [5]. Because of its slightly longer exposure time, multidetector CT (MDCT) has a slightly higher effective radiation dose of 1.0 to $1.5 \mathrm{mSv}$ in men and 1.1 to $1.9 \mathrm{mSv}$ in women. Our respondents were asked if the benefit of an indicated CAC scan outweighed a 1-mSv exposure to radiation and the associated carcinogenic potential. While $60 \%$ believed that the benefit was enough to outweigh the risk, $40 \%$ (12 of 30) respondents felt otherwise. Such a discernible split on a key question regarding the risk-benefit ratio on CAC scans will surely impact the adoption of this technology. Annual background radiation exposure from natural sources usually ranges from 1.5 to $3.5 \mathrm{mSv}$ and can go up to $50 \mathrm{mSv}$. Moreover, using the relative risk reduction with $20 \mathrm{mg}$ of rosuvastatin, the five-year number needed to treat (NNT) to prevent one cardiovascular event varies from 124 for those with a CAC score of 0 to 19 for those with a CACS above 100. Hence, identifying 19 patients with a CAC score above 100 and risk stratifying them to a more targeted therapy can prevent a major cardiovascular event at the cost of one-time exposure to $1 \mathrm{mSv}$ of radiation.

One of the major obstacles to greater adoption of CAC scores as a screening modality pertains to issues with pre-approval from insurance agencies. A typical CAC screening costs 500 dollars, with MDCT being generally less expensive than EBCT [25]. If insurance refuses coverage or asks for a hectic pre-approval process, physicians are likely to get discouraged and avoid a modality. Other options are to ask patients to pay outof-pocket, which again adds more workload to a provider and more cost to the patient. Figure 7 shows that only $10 \%$ of respondents reported to have "never" encountered such problems, while a staggering $70 \%$ of respondents to have encountered issues with insurance with varying frequencies. Issues with insurance approval were hence identified as a major obstacle in higher adoption of this modality.

In the last question on the survey, which was meant to investigate the issues perceived as major obstacles to a greater adoption of CAC scans, $70 \%$ of respondents reported that lack of enthusiasm/adoption among providers was the biggest obstacle to higher usage (Figure 8). In face of such an evident divide within endusers and providers within a singular healthcare system, on every aspect of the test, from its indications to its financial and radiation costs, it is not hard to imagine the lack of enthusiasm surrounding its adoption. Moreover, it is a fact that the niche populations that derive maximum benefits from the screen are small subsets that need to be appropriately identified for the sake of patient benefit as well as insurance approval. When intellectual dissonance on a subject is compounded by approval problems from insurance agencies, it is only natural that most providers would be inclined to use simpler risk factor based tools alone to arrive at their decisions.

\section{Recommendations}

1. For trainees who are still undergoing training in internal medicine and cardiology, there should be a seminar or workshop geared to better equip these future practitioners towards the usage of this useful tool. For those who are currently in practice, educational online modules and training workshops with CME (Continuing Medical Education) credits can be offered to help refresh salient applications of CAC scanning. 
Moreover, primary care offices can be provided with posters of simplified decision-making algorithms and charts to facilitate appropriate ordering.

2. A rectification of misconceptions regarding cost-effectiveness and radiation exposure can be achieved by embedding modules on downstream testing as well as risk-benefit ratios in terms of radiation exposure within the aforementioned training workshops and online modules.

3. To address the difficulty with insurance pre-approval for these scans, it would be productive to reach out to physicians that have had higher success in achieving approval for their ordered CAC scan and recruiting them to offer insights during workshops and seminars to their colleagues. Such pioneers can also lead quality improvement initiatives for ancillary staff in outpatient offices where such tests are often ordered. If improved patient selection by providers is accompanied by appropriate documentation by ancillary staff, the resultant improved approval from insurance can itself be a good stimulus for increased adoption of this scan.

4. The biggest limitation to higher adoption of CAC scans as a screening tool seems to be a lack of enthusiasm among providers. The best method to stimulate enthusiasm among physicians is to show results. Compiling a system-wide registry of all CAC scans conducted and resultant re-stratification can generate more interest among providers.

\section{Conclusions}

CAC scoring is a viable coronary atherosclerotic screening modality that can be used to great effect in asymptomatic adults if ordering physicians are well-trained in patient selection. In addition to identifying patients who need to be worked up further, it can also help re-stratify patients within-risk groups and inform decision-making regarding the use of lipid-lowering therapies. Many of the obstacles in adoption of this screening modality can be overcome by focused training modules, educational seminars, and appropriate documentation. A concerted quality improvement initiative can achieve dramatic improvements in appropriate adoption of this technology.

\section{Additional Information \\ Disclosures}

Human subjects: All authors have confirmed that this study did not involve human participants or tissue. Animal subjects: All authors have confirmed that this study did not involve animal subjects or tissue. Conflicts of interest: In compliance with the ICMJE uniform disclosure form, all authors declare the following: Payment/services info: All authors have declared that no financial support was received from any organization for the submitted work. Financial relationships: All authors have declared that they have no financial relationships at present or within the previous three years with any organizations that might have an interest in the submitted work. Other relationships: All authors have declared that there are no other relationships or activities that could appear to have influenced the submitted work.

\section{References}

1. Nasir K, Clouse M: Role of nonenhanced multidetector CT coronary artery calcium testing in asymptomatic and symptomatic individuals. Radiology. 2012, 264:637-49. 10.1148/radiol.12110810

2. Greenland P, Smith SC Jr, Grundy SM: Improving coronary heart disease risk assessment in asymptomatic people: role of traditional risk factors and noninvasive cardiovascular tests. Circulation. 2001, 104:1863-7. 10.1161/hc4201.097189

3. Kannel WB, Dawber TR, Kagan A, Revotskie N, Stokes J 3rd: Factors of risk in the development of coronary heart disease--six year follow-up experience. The Framingham Study. Ann Intern Med. 1961, 55:33-50. 10.7326/0003-4819-55-1-33

4. Wilson PW, D'Agostino RB, Levy D, Belanger AM, Silbershatz H, Kannel WB: Prediction of coronary heart disease using risk factor categories. Circulation. 1998, 97:1837-47. 10.1161/01.cir.97.18.1837

5. Budoff MJ, Achenbach S, Blumenthal RS, et al.: Assessment of coronary artery disease by cardiac computed tomography: a scientific statement from the American Heart Association Committee on Cardiovascular Imaging and Intervention, Council on Cardiovascular Radiology and Intervention, and Committee on Cardiac Imaging, Council on Clinical Cardiology. Circulation. 2006, 114:1761-91. 10.1161/CIRCULATIONAHA.106.178458

6. Li H, Hong S, Qian J, Zheng Y, Yang J, Yi O: Cross talk between the bone and immune systems: osteoclasts function as antigen-presenting cells and activate CD4+ and CD8+ T cells. Blood. 2010, 116:210-7. 10.1182/blood-2009-11-255026

7. Tintut Y, Parhami F, Tsingotjidou A, Tetradis S, Territo M, Demer LL: 8-Isoprostaglandin E2 enhances receptor-activated NFkappa B ligand (RANKL)-dependent osteoclastic potential of marrow hematopoietic precursors via the cAMP pathway. J Biol Chem. 2002, 277:14221-6. 10.1074/jbc.M111551200

8. Bear M, Butcher M, Shaughnessy SG: Oxidized low-density lipoprotein acts synergistically with betaglycerophosphate to induce osteoblast differentiation in primary cultures of vascular smooth muscle cells. J Cell Biochem. 2008, 105:185-93. 10.1002/jcb.21812

9. Bild DE, Bluemke DA, Burke GL, et al.: Multi-Ethnic Study of Atherosclerosis: objectives and design . Am J Epidemiol. 2002, 156:871-81. 10.1093/aje/kwf113

10. Schmermund A, Möhlenkamp S, Stang A, et al.: Assessment of clinically silent atherosclerotic disease and 
established and novel risk factors for predicting myocardial infarction and cardiac death in healthy middleaged subjects: rationale and design of the Heinz Nixdorf RECALL Study. Risk Factors, Evaluation of Coronary Calcium and Lifestyle. Am Heart J. 2002, 144:212-8. 10.1067/mhj.2002.123579

11. Oei HH, Vliegenthart R, Hak AE, Iglesias del Sol A, Hofman A, Oudkerk M, Witteman JC: The association between coronary calcification assessed by electron beam computed tomography and measures of extracoronary atherosclerosis: The rotterdam coronary calcification study. J Am Coll Cardiol. 2002, 39:174551. 10.1016/S0735-1097(02)01853-3

12. Hoffmann U, Massaro JM, Fox CS, Manders E, O'Donnell CJ: Defining normal distributions of coronary artery calcium in women and men (from the Framingham Heart Study). Am J Cardiol. 2008, 102:1136-41. 10.1016/j.amjcard.2008.06.038

13. Agatston AS, Janowitz WR, Hildner FJ, Zusmer NR, Viamonte M Jr, Detrano R: Quantification of coronary artery calcium using ultrafast computed tomography. J Am Coll Cardiol. 1990, 15:827-32. 10.1016/07351097(90)90282-t

14. Sarwar A, Shaw LJ, Shapiro MD, et al.: Diagnostic and prognostic value of absence of coronary artery calcification. JACC Cardiovasc Imaging. 2009, 2:675-88. 10.1016/j.jcmg.2008.12.031

15. Blaha M, Budoff MJ, Shaw LJ, et al.: Absence of coronary artery calcification and all-cause mortality . JACC Cardiovasc Imaging. 2009, 2:692-700. 10.1016/j.jcmg.2009.03.009

16. Okwuosa TM, Greenland P, Ning H, Liu K, Lloyd-Jones DM: Yield of screening for coronary artery calcium in early middle-age adults based on the 10-year Framingham Risk Score: the CARDIA study. JACC Cardiovasc Imaging. 2012, 5:923-30. 10.1016/j.jcmg.2012.01.022

17. Okwuosa TM, Greenland P, Ning H, et al.: Distribution of coronary artery calcium scores by Framingham 10year risk strata in the MESA (Multi-Ethnic Study of Atherosclerosis) potential implications for coronary risk assessment. J Am Coll Cardiol. 2011, 57:1838-45. 10.1016/j.jacc.2010.11.053

18. Criqui MH, Denenberg JO, Ix JH, et al.: Calcium density of coronary artery plaque and risk of incident cardiovascular events. JAMA. 2014, 311:271-8. 10.1001/jama.2013.282535

19. Liu M, Wronski L: Examining completion rates in web surveys via Over 25,000 real-world surveys . Social Sci Comp Rev. 2017, 36:116-24. 10.1177/0894439317695581

20. Lloyd-Jones DM, Braun LT, Ndumele CE, Smith SC Jr, Sperling LS, Virani SS, Blumenthal RS: Use of risk assessment tools to guide decision-making in the primary prevention of atherosclerotic cardiovascular disease: a special report from the American Heart Association and American College of Cardiology. Circulation. 2019, 139:1162-77. 10.1161/CIR.0000000000000638

21. Lakoski SG, Greenland P, Wong ND, et al.: Coronary artery calcium scores and risk for cardiovascular events in women classified as "low risk" based on Framingham risk score: the Multi-Ethnic Study of Atherosclerosis (MESA). Arch Intern Med. 2007, 167:2437-42. 10.1001/archinte.167.22.2437

22. Detrano R, Yiannikas J, Salcedo EE, Rincon G, Go RT, Williams G, Leatherman J: Bayesian probability analysis: a prospective demonstration of its clinical utility in diagnosing coronary disease. Circulation. 1984, 69:541-7. 10.1161/01.cir.69.3.541

23. Bonow RO: Should coronary calcium screening be used in cardiovascular prevention strategies? . N Engl J Med. 2009, 361:990-7. 10.1056/NEJMcp0902177

24. Rozanski A, Gransar H, Shaw LJ, et al.: Impact of coronary artery calcium scanning on coronary risk factors and downstream testing the EISNER (Early Identification of Subclinical Atherosclerosis by Noninvasive Imaging Research) prospective randomized trial. J Am Coll Cardiol. 2011, 57:1622-32. 10.1016/j.jacc.2011.01.019

25. Kim KP, Einstein AJ, Berrington de González A: Coronary artery calcification screening: estimated radiation dose and cancer risk. Arch Intern Med. 2009, 169:1188-94. 10.1001/archinternmed.2009.162 